\title{
Coverage-dependent adsorption model of atomic oxygen on $\mathrm{Ag}(111)$
}

\author{
Carson J. Mize, ${ }^{1}$ Sara B. Isbill,,${ }^{1}$ a) and Sharani Roy ${ }^{1, b}$ ) \\ Department of Chemistry, University of Tennessee, Knoxville, \\ TN 37996
}

(Dated: 21 July 2021)

Theoretical gas-surface models that describe adsorption over a wide range of coverages can provide qualitative insight into chemical phenomena that occur at intermediate to high coverages, such as subsurface adsorption, surface reconstruction, and industrial heterogeneous catalysis. However, most atomistic, quantum-mechanical models of gas-surface adsorption are limited to low adsorbate coverage due to the large computational cost of models built using many surface atoms and adsorbates. To investigate adsorption in the subsurface of a crystalline solid at high coverage, we present a lattice-gas adsorption model that includes surface and subsurface sites of the solid, and is fully parametrized using density functional theory. We apply the model to study the coverage-dependent adsorption of atomic oxygen on the $\mathrm{Ag}(111)$ surface. Oxygen population distributions calculated using the model show the onset of subsurface adsorption at a total coverage of approximately $\frac{1}{4}$ monolayer, the saturation of surface adsorption at a total coverage of approximately $\frac{1}{3}$ monolayer, and a greater accumulation of oxygen in the second rather than the first subsurface at total coverages greater than $\frac{1}{2}$ monolayer. Computation of core-electron binding energies and projected density of states of an oxygen distribution predicted by the model reveal qualitative differences in oxygen-silver bonding at the surface and subsurface, suggesting that oxygen adsorbed in the two regions could play distinct roles in surface chemistry.

Keywords: atomic oxygen, $\operatorname{Ag}(111)$, density functional theory, adsorption, subsurface, lattice-gas model, pairwise interactions, core-electron binding energies, projected density of states.

\footnotetext{
a) Present affiliation: Oak Ridge National Laboratory, Oak Ridge, TN 37830.

b) Electronic mail: sharani.roy@utk.edu
} 


\section{INTRODUCTION}

The adsorption of an atom or molecule on a solid surface is an elementary step of diverse chemical processes at surfaces, ranging from corrosion, gas storage, and heterogeneous catalysis, to chemical sensing and formation of self-assembled monolayers. While advances in periodic density functional theory (DFT) have enabled the quantum-mechanical computation of adsorption properties at low adsorbate concentrations with high accuracy and efficiency, large adsorbate concentrations remain a challenge to compute due to their complex surface chemistry and steep computational cost. The cost can be kept low by keeping the number of adsorbates fixed and decreasing the lateral dimensions of the supercell, but the coverages constructed in this matter enforce highly periodic adlayer structures. Chemically meaningful, flexible, yet efficient theoretical models capable of describing adsorption over a wide range of concentration can provide mechanistic insight into processes, such as surface reconstruction, surface oxidation, and industrial heterogeneous catalysis.

Lattice-gas models and cluster-expansion methods are powerful theoretical approaches to study coverage-dependent adsorption and the effects of inter-adsorbate interactions on adsorption as coverage is increased. These approaches describe the total adsorption energy as the sum of non-interacting energies of the adsorbates at different lattice sites plus $n$-body

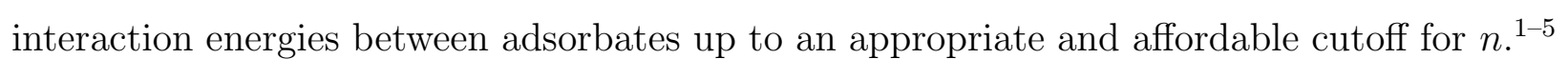
Some recent examples in which the site-adsorption energies and interaction energies were calculated using DFT include coverage-dependent adsorption studies of hydrogen on Fe(100) and atomic oxygen on $\mathrm{Pt}(111)$, and lateral adsorbate interactions in the NO-CO reaction system on $\operatorname{Rh}(100)$ and $\operatorname{Rh}(111) \cdot[6]$ Cluster-expansion methods are also used in conjunction with Monte Carlo and kinetic Monte Carlo simulations, such as in the studies of adlayer phases in $\mathrm{H} / \mathrm{Cu}(100), \mathrm{H} / \mathrm{Ag}(100)$, and $\mathrm{O} / \mathrm{Cu}(100)$, and ethylene epoxidation on $\mathrm{Ag}(100)$, $\operatorname{Ag}(110)$, and $\operatorname{Ag}(111) .910$

The examples of adsorption models of atomic oxygen on metal surfaces cited above are but a few from the vast literature developed over several decades to elucidate the mechanisms of oxidation of metals $\frac{1112}{12}$ Among these systems, the interaction of atomic oxygen with silver surfaces has been widely investigated due to the efficacy of silver as an indus-

trial oxidation catalyst for the conversion of ethylene to ethylene oxide and methanol to formaldehyde. $10 \mid 13$ Evidence from several experimental studies strongly indicates that as 
the surface concentration of atomic oxygen increases, it percolates to the subsurface region, i.e., the region just below the surface of the metal. ${ }^{26} \sqrt{32}$ Subsurface adsorption has been explored in many gas-surface systems, such as $\mathrm{CO}_{2} / \mathrm{Cu}(100)$ and $\mathrm{Cu}(111), \mathrm{H}_{2} / \mathrm{Ni}(111)$, and $\mathrm{O} / \mathrm{Al}(111) \stackrel{33}{[35}$ Results suggest that subsurface adsorbates play an important role in surface reconstruction and catalysis, such as in the case of oxygen covered silver surfaces, but

the exact nature of their participation is not yet completely understood. ${ }^{26[27 \mid 36} \sqrt[45]{4}$ Therefore, atomistic models that include surface and subsurface sites can promote a deeper fundamental understanding of the nature and prevalence of subsurface adsorption in surface chemistry.

To study the interplay between binding of gaseous species above and below the surface of a crystalline solid, we have developed a lattice-gas adsorption model of atomic oxygen on $\operatorname{Ag}(111)$ that includes surface and subsurface sites, and is fully parameterized using DFT. To our knowledge, this is the first lattice-gas adsorption model that describes both surface and subsurface adsorption. The model describes oxygen adsorption at the most strongly binding sites, but can be extended to include all types of surface and subsurface sites on $\operatorname{Ag}(111)$. The effects of coverage are described using pairwise interactions between oxygen atoms co-adsorbed on different sites of the metal. Using this model, we computed oxygen adsorption energies and population distributions on the surface and subsurface of $\mathrm{Ag}(111)$ as a function of total coverage. Based on the predicted population distribution, we constructed a DFT model of a total coverage of $\frac{1}{2}$ monolayer (ML) to study the differences in electronic properties of atomic oxygen at the surface and subsurface. Our study demonstrates that the lattice-gas adsorption model provides a simple theoretical framework to explore the competition between surface and subsurface adsorption in gas-surface systems.

\section{METHOD}

\section{A. Lattice-gas adsorption model}

In our lattice-gas adsorption model of atomic oxygen on $\operatorname{Ag}(111)$, the total adsorption energy $E_{\text {total }}$ of $N$ oxygen atoms is defined as:

$$
\begin{aligned}
E_{\text {total }}(N) & =\sum_{i=1}^{N} E_{i}+\sum_{i=1}^{N} \sum_{j>i}^{N} \Delta E_{i j} \\
\Delta E_{i j} & =E_{i j}-E_{i}-E_{j}
\end{aligned}
$$


where $E_{i}\left(E_{j}\right)$ is the adsorption energy of the $i$-th $(j$-th) oxygen atom in the absence of any neighboring oxygen and $E_{i j}$ is the total adsorption energy of the $i$-th and $j$-th oxygen atoms when both are bound to $\operatorname{Ag}(111)$. Therefore, $\Delta E_{i j}$ is the pair-interaction energy between the $i$-th and $j$-th adsorbed oxygen atoms. $\Delta E_{i j}$ is positive for attractive or cooperative interactions, negative for repulsive or uncooperative interactions, and set to zero for a pair of oxygen atoms separated by longer than third-neighbor distances. The values of $E_{i}$ and $E_{i j}$ were calculated using DFT, as described in Section IIB.

\section{B. DFT calculations}

DFT calculations were performed using the revised Perdew, Burke, and Ernzerhof with Pade Approximation (RPBE) $\underline{46-48}$ exchange-correlation functional as implemented within the Vienna ab initio Simulation Package (VASP) $\underline{49} \underline{52}$ An energy cutoff of $400 \mathrm{eV}$ was used for the plane-wave basis set, and interactions between nuclei and core electrons were described using the projector augmented wave method ${ }^{53 \mid 54}$ The Methfessel-Paxton ${ }^{55}$ smearing method was applied with a smearing width of $0.2 \mathrm{eV}$, giving an entropy change less than $0.01 \mathrm{meV} /$ atom. The RPBE functional, combined with a $15 \times 15 \times 15$ Monkhorst-Pack 56 $k$-point grid gave a bulk lattice constant of $4.213 \AA$ for $\mathrm{Ag}$, which is $3.0 \%$ greater than the corresponding experimental value of $4.09 \AA .57$

The $\operatorname{Ag}(111)$ surface was modeled using a $\mathrm{p}(3 \times 4)$ supercell with six layers of $\mathrm{Ag}$ atoms and a vacuum layer twice as thick as the surface slab along the $z$-direction, as shown in Figure 1. The dimensions of the supercell were $8.93 \AA \times 10.32 \AA \times 43.78 \AA$. A $5 \times 5 \times 1$ Monkhorst-Pack ${ }^{\sqrt{56}} k$-point grid was used to sample the first Brillouin zone. The bottom three layers of the supercell were fixed at the bulk crystal geometry, whereas the top three layers and all adsorbates were allowed to relax in geometry optimizations until the forces on the mobile nuclei were less than $0.05 \mathrm{eV} / \AA$.

The adsorption energy of an oxygen atom on $\operatorname{Ag}(111), E_{i}$, is defined as

$$
E_{i}=-\left(E_{O / A g(111)}-E_{A g(111)}-E_{O}\right)
$$

where $E_{O / A g(111)}$ is the energy of the relaxed $\mathrm{O} / \mathrm{Ag}(111)$ supercell, $\mathrm{E}_{A g(111)}$ is the energy of the relaxed $\operatorname{Ag}(111)$ supercell, and $\mathrm{E}_{O}$ is the energy of an isolated oxygen atom placed inside the empty supercell. Based on the definition, a positive adsorption energy indicates stable 
(a)

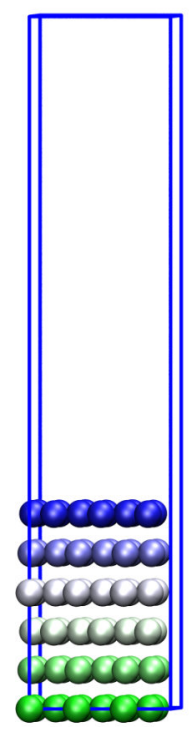

(b)

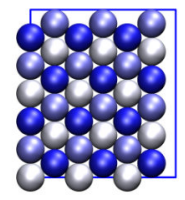

(c)

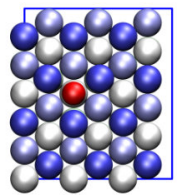

(d)

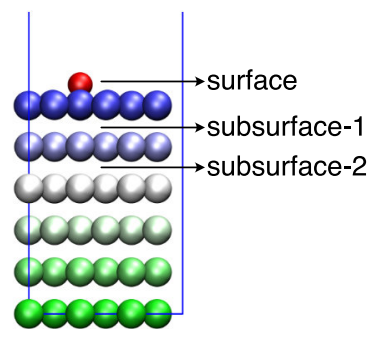

FIG. 1. (a) and (b): Side and top views, respectively, of the $\operatorname{Ag}(111)$ supercell with $\mathrm{Ag}$ atoms colored by layer, starting from dark blue in the topmost layer to bright green in the bottom layer. The top view shows the three-fold symmetry of the surface and the ABC stacking of the layers. (c) and (d): Top and side views, respectively, of an oxygen atom (red) adsorbed on a face-centeredcubic hollow site of the $\operatorname{Ag}(111)$ supercell. The surface, first subsurface ("subsurface-1"), and second subsurface ("subsurface-2") regions of the supercell are labelled in the side view.

adsorption. If there are $N>1$ oxygen atoms in the supercell, the formula is adjusted to:

$$
E_{i}=\frac{-\left(E_{(N \times O) / A g(111)}-E_{A g(111)}-N \times E_{O}\right)}{N}
$$

The fractional oxygen coverage $(\theta)$ was defined as $\frac{N}{N_{\text {Ag,surface }}}$, where $N_{\text {Ag,surface }}$ is the number of Ag atoms on the surface. Since the supercell has $12 \mathrm{Ag}$ atoms in each layer, the lowest investigated coverage was $\frac{1}{12} \approx 0.08 \mathrm{ML}$. The energies were corrected for the dipole formed due to charge transfer from the surface to the adsorbates. Charges on Ag and O atoms were calculated using the Bader method of charge density partitioning. $.58,60$ The relative shifts in binding energies of the $1 s$ core electrons of adsorbed oxygen were calculated by exciting the $\mathrm{O}_{1 s}$ electron into the valence using the method implemented in VASP ${ }^{61} 63$ The values were averaged over the relevant oxygen atoms to study the effects of coverage on the core-electron binding energies. 
TABLE I. Adsorption energy $\left(E_{a d s}\right)$, perpendicular atom-surface distance $\left(z_{O-A g(111}\right)$, and Bader charge $\left(q_{O}\right)$ of atomic oxygen adsorbed to $\mathrm{Ag}(111)$ at $\theta=\frac{1}{12} \mathrm{ML}$.

\begin{tabular}{|c|c|c|c|c|}
\hline Region & Adsorption site & $E_{a d s}(\mathrm{eV})$ & $z_{O-A g(111)}(\AA)$ & $q_{O}\left(e^{-}\right)$ \\
\hline surface & fcc hollow & 3.52 & 1.17 & -0.9 \\
\hline surface & hcp hollow & 3.43 & 1.23 & -0.9 \\
\hline surface & bridge & 3.23 & 1.24 & -0.9 \\
\hline surface & top & 2.13 & 1.85 & -0.7 \\
\hline first subsurface & octahedral & 3.03 & -0.57 & -1.0 \\
\hline first subsurface & tetrahedral & 2.55 & -1.29 & -0.9 \\
\hline second subsurface & octahedral & 2.73 & -3.56 & -1.0 \\
\hline second subsurface & tetrahedral & 2.48 & -3.88 & -1.0 \\
\hline second subsurface & inverted tetrahedral & 2.42 & -2.93 & -1.0 \\
\hline
\end{tabular}

\section{RESULTS AND DISCUSSION}

\section{A. Adsorption at low coverage}

Table 1 shows the adsorption energy, Bader charge, and perpendicular atom-surface distance of $\mathrm{O}$ chemisorbed to high-symmetry sites on the surface and in the subsurface of $\operatorname{Ag}(111)$ at a coverage of $\frac{1}{12} \mathrm{ML}$. The surface sites are face-centered cubic (fcc) hollow, hexagonal close-packed (hcp) hollow, bridge, and top; and the subsurface sites are octahedral, tetrahedral, and inverted-tetrahedral. Images of the binding sites are shown in Section S1 of the Supplementary Information (SI). Two subsurface regions were included in our study, the region between the first and second layers of metal atoms, hereafter referred to as the first subsurface, and the region between the second and third layers of metal atoms, hereafter referred to as the second subsurface. While past studies have computed adsorption energies of atomic oxygen in the first subsurface of $\operatorname{Ag}(111)$, 36]38 41/44 our study is the first to study adsorption in the second subsurface using DFT and include it in a lattice-gas model. The depth of the oxygen atoms shown in Table $\mathbb{1}$ suggests that oxygen in the second subsurface could be detected in surface-sensitive experiments, such as X-ray photoelectron 


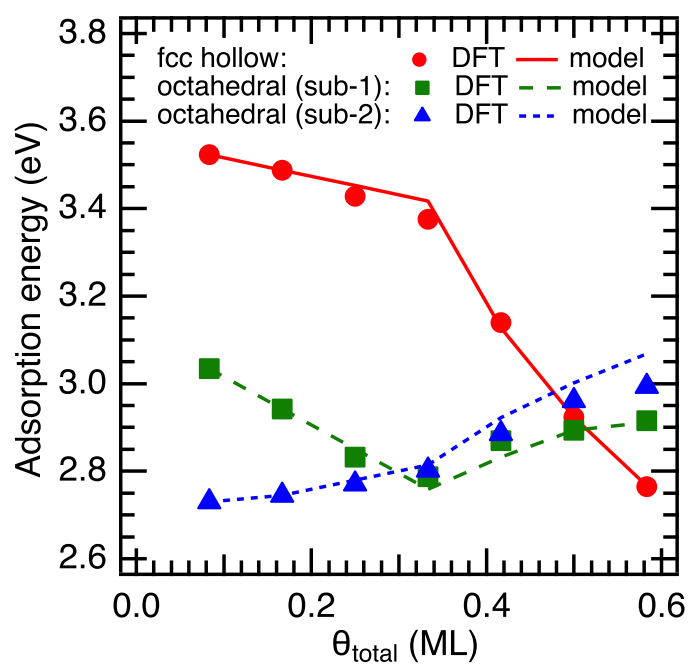

FIG. 2. Adsorption energy of atomic oxygen at the fcc-hollow site on the surface, octahedral site in the first subsurface ("sub-1"), and octahedral site in the second subsurface ("sub-2") of $\operatorname{Ag}(111)$ as a function of total oxygen coverage $\left(\theta_{\text {total }}\right)$.

spectroscopy (XPS) $\underline{27}$

O binds most strongly to the fcc hollow site on the surface and to the octahedral sites in the subsurface. This site preference agrees with previous studies reported in the literature. $\stackrel{3738}{38}$ The calculated adsorption energy of $3.52 \mathrm{eV}$ at the fcc hollow site agrees well with adsorption energy of $3.45 \mathrm{eV}$ extracted from temperature-programmed desorption experiments. ${ }^{30}$ The inverted tetrahedral site in the first subsurface is not included in the table, because at this coverage, the oxygen atom rises to the surface and nestles in the hcp hollow site. Overall, our results show that at the low coverage of $\frac{1}{12} \mathrm{ML}$, O binds more strongly to the surface than to the subsurface. The Bader charges show that 0.7-1.0 electron is transferred from the metal surface to the adsorbed oxygen. This charge transfer has a strong effect on the adsorption behavior at high coverages, as described in Section IIIB.

\section{B. Effects of coverage on adsorption energy}

Figure 2 shows the adsorption energy of atomic oxygen on $\operatorname{Ag}(111)$ as a function of total coverage $\left(\theta_{\text {total }}\right)$ at the most strongly binding sites, calculated using DFT (points) and the adsorption model (lines). While the adsorption energy decreases with coverage at fcc hollow 
sites, it increases with coverage at the octahedral sites in the second subsurface. At the octahedral sites in the first subsurface, the adsorption energy decreases with coverage in the range, $\theta_{\text {total }}<\frac{1}{3} \mathrm{ML}$ and increases with coverage in the range, $\theta_{\text {total }}>\frac{1}{3} \mathrm{ML}$. Due to the differences in coverage effects between the surface and subsurface, atomic oxygen becomes more strongly bound to the subsurface than to the surface for $\theta_{\text {total }}>\frac{1}{2}$ ML. The adsorption energy at the hcp hollow site varies similarly as that at the fcc hollow site; however, adsorption at the bridge and top sites is not stable at higher coverages. In both the first and second subsurface regions, the adsorption energy at the tetrahedral and inverse tetrahedral sites varies similarly as to the octahedral site. Further, we found that the adsorption of atomic oxygen to the inverted tetrahedral site in the first subsurface becomes stable at higher coverages.

The gradient of adsorption energy with coverage in both surface and subsurface regions shows a sudden change when $\theta_{\text {total }}$ exceeds $\frac{1}{3}$ ML. This change occurs because coverages greater than $\frac{1}{3}$ ML necessarily have at least one same-site first-neighbor O-O interaction due to the symmetry of the $\operatorname{Ag}(111)$ lattice. The pairwise interaction energies from our model show that whereas the first-neighbor interaction is strongly repulsive between fcc hollow sites $(-0.532 \mathrm{eV})$, it is weakly attractive between octahedral sites in the first subsurface $(+0.076$ $\mathrm{eV})$, and moderately attractive between octahedral sites in the second subsurface $(+0.181$ $\mathrm{eV}$ ). The second-neighbor interactions, which contribute the most to adsorption energies at low coverage, are repulsive at fcc hollow sites $(-0.035 \mathrm{eV})$ as well as octahedral sites in the first subsurface $(-0.092 \mathrm{eV})$, but attractive at octahedral sites in the second subsurface $(+0.034 \mathrm{eV})$, thus explaining the variations in adsorption energy at coverage less than than $\frac{1}{3} \mathrm{ML}$.

Figure 2 shows that our adsorption model reproduces the DFT-computed adsorption energies as well as their variations with surface and subsurface coverage with good accuracy. A table of model parameters and further comparisons between DFT-computed and modelcomputed adsorption energies are shown in Sections S2 and S3 of the SI.

\section{Atomic oxygen distributions as a function of total coverage}

While DFT calculations become expensive with increasing coverage, especially when atomic oxygen occupies more than one type of surface site, our model can efficiently calcu- 


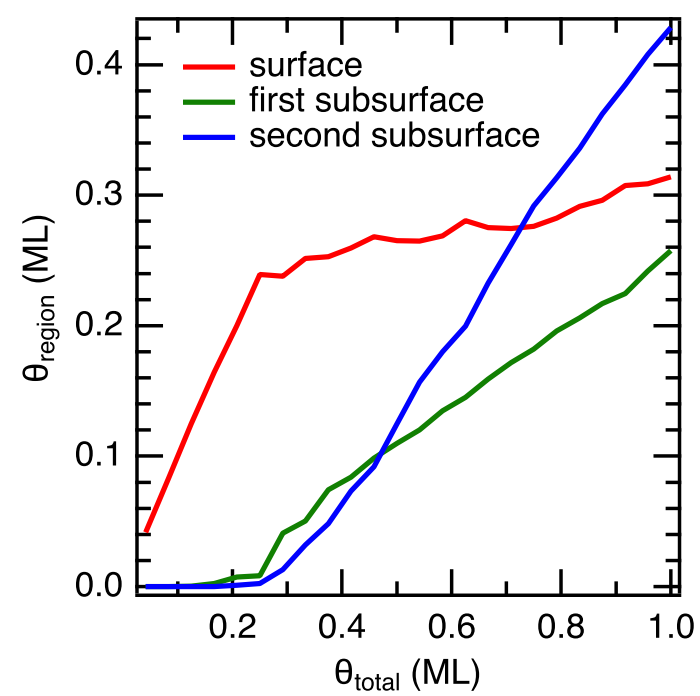

FIG. 3. Boltzmann-averaged coverage of atomic oxygen on the surface, first subsurface, and second subsurface of $\operatorname{Ag}(111)$ as function of total coverage at room temperature.

late the adsorption energy of any coverage of atomic oxygen occupying any site on $\operatorname{Ag}(111)$, provided that type of site is included in the model. In the present study, our adsorption model includes the most favored surface and subsurface sites shown in Table II, i.e., the fcc hollow and hcp hollow sites on the surface, and the octahedral sites in the first and second subsurface regions. All the parameters of the model are presented in Section S2 of the SI. Using this model, we calculated the Boltzmann-averaged coverage distribution of atomic oxygen on $\operatorname{Ag}(111)$ at the four sites as a function of total coverage, as shown in Figure 3 . The distributions were calculated at a surface temperature of $298.15 \mathrm{~K}$. A key difference between Figures 2 and 3 is that a specific total coverage in Figure 2 was created by binding oxygen atoms to a single type of site, whereas a total coverage in Figure 3 was created by allowing oxygen to bind to any of the four types of sites included in the model. Further, the binding sites in Figure 2 were chosen to maximize the distance between neighboring oxygen atoms for a specific total coverage, whereas the binding sites in Figure 3 were randomly chosen from all available sites and the resulting oxygen arrangements were used to calculate the Boltzmann-averaged population distribution at each chosen total coverage.

The calculated oxygen population distribution in Figure 3 shows that for $\theta_{\text {total }}<\frac{1}{4}$ ML, atomic oxygen adsorbs to the fcc hollow and hcp hollow sites on the surface, whereas between 


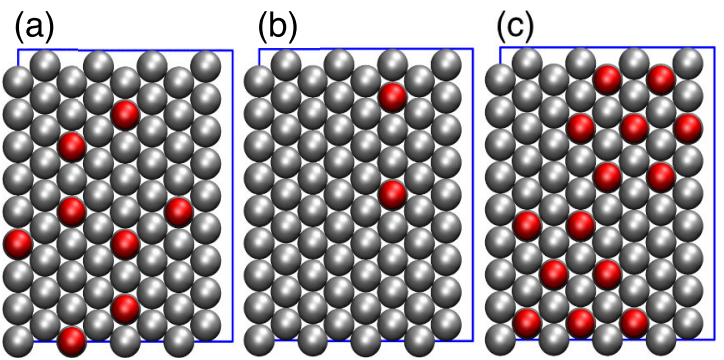

FIG. 4. Top views of oxygen atoms adsorbed to the (a) surface, (b) first subsurface, and (c) second subsurface of $\mathrm{Ag}(111)$ from an energetically favorable population distribution predicted by the model. The total oxygen coverage is $1 \mathrm{ML}$, occupying a $\mathrm{p}(4 \times 6)$ supercell of $\mathrm{Ag}(111)$. The regional coverages are $\frac{1}{3}$ ML on the surface, $\frac{1}{12}$ ML in the first subsurface, and $\frac{7}{12} \mathrm{ML}$ in the second subsurface. Each image shows only the oxygen atoms in that region and omits the silver atoms above that region for clarity. All silver atoms are colored grey and oxygen atoms are colored red.

$\frac{1}{4}<\theta_{\text {total }}<\frac{1}{2} \mathrm{ML}$, the surface oxygen coverage remains close to $\frac{1}{4} \mathrm{ML}$, and the excess oxygen distributes about equally between the two subsurface regions. In the range, $\frac{1}{2}<\theta_{\text {total }}<1$ ML, the surface oxygen coverage slowly approaches $\frac{1}{3} \mathrm{ML}$, and the excess oxygen accumulates more in the second subsurface than in the first subsurface due to more strongly cooperative O-O first-neighbor interactions. As a result, in the Boltzmann-averaged regional coverages at $\theta_{\text {total }}=1 \mathrm{ML}, 0.31 \mathrm{ML}$ of atomic oxygen adsorbs to the surface, 0.26 ML adsorbs to the first subsurface, and $0.43 \mathrm{ML}$ adsorbs to the second subsurface of $\mathrm{Ag}(111)$.

In the lowest-energy geometries of $\mathrm{O} / \mathrm{Ag}(111)$ obtained from the model at $\theta_{\text {total }}>\frac{1}{2}$ ML, atomic oxygen uniformly distributes between fcc hollow and hcp hollow sites on the surface in order to minimize first-neighbor O-O interactions, whereas it forms islands in the second subsurface in order to maximize first-neighbor O-O interactions. An example of a highly energetically stable oxygen distribution when the model was implemented on a $\mathrm{p}(4 \times 6) \mathrm{Ag}(111)$ supercell is shown in Figure 4. The inter-site interaction energies also play an important role in increasing the atomic oxygen populations in the second compared to the first subsurface at $\theta_{\text {total }}>\frac{1}{2} \mathrm{ML}$. All the O-O interaction energies between the fcc hollow sites and the octahedral sites in the first subsurface, particularly the first-neighbor interaction energy $(-0.573 \mathrm{eV})$, are repulsive. The interaction energies between oxygen atoms at the hcp hollow sites and the octahedral sites in the first subsurface, except for a moderately attractive 


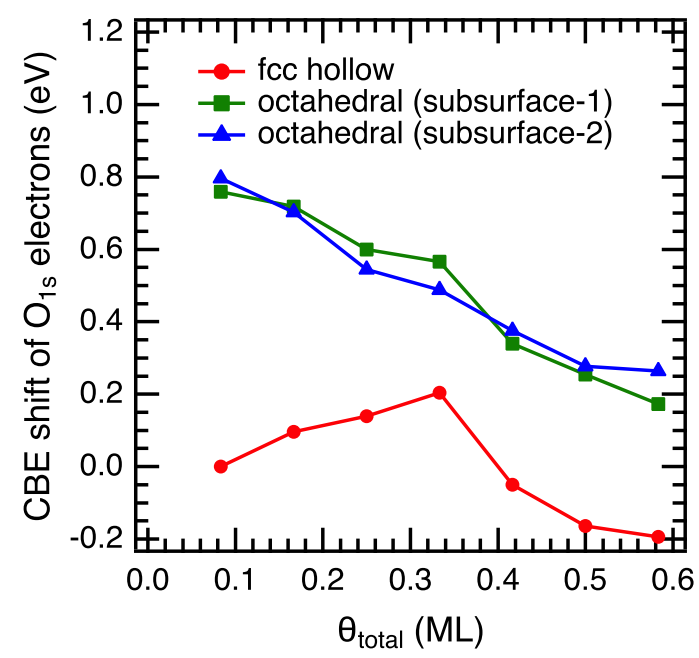

FIG. 5. CBE shifts of 1s electrons of atomic oxygen at the fcc-hollow site on the surface, octahedral site in the first subsurface ("sub-1"), and octahedral site in the second subsurface ("sub-2") of $\operatorname{Ag}(111)$ as a function of total oxygen coverage $\left(\theta_{\text {total }}\right)$. The oxygen coverages and arrangements are the same as used in Figure 2.

second neighbor interaction $(+0.180 \mathrm{eV})$, are also repulsive. In contrast, oxygen atoms at the octahedral sites in the second subsurface have a combination of weakly attractive and weakly repulsive interactions with oxygen atoms at either of the two surface sites and with oxygen atoms at the octahedral sites in the first subsurface. Consequently, it is energetically more favorable for atomic oxygen to adsorb to the second surface than to the first subsurface at high total coverages.

\section{Electronic and bonding properties of surface oxygen and subsurface oxygen}

To probe the differences in electronic properties between adsorbates on the surface and adsorbates in the subsurface, we calculated the core-electron binding energies (CBE) of oxygen atoms at fcc hollow sites on the surface, octahedral sites in the first subsurface, and octahedral sites in the second subsurface of $\mathrm{Ag}(111)$ as a function of coverage using DFT, as shown in Figure 5. The CBE of an adsorbed oxygen atom increases when its $1 s$ electrons are less shielded from the nucleus by the $2 s$ and $2 p$ electrons, indicating that the valence electrons are more strongly shared with neighboring nuclei. The coverages and oxygen arrangements 
(a)

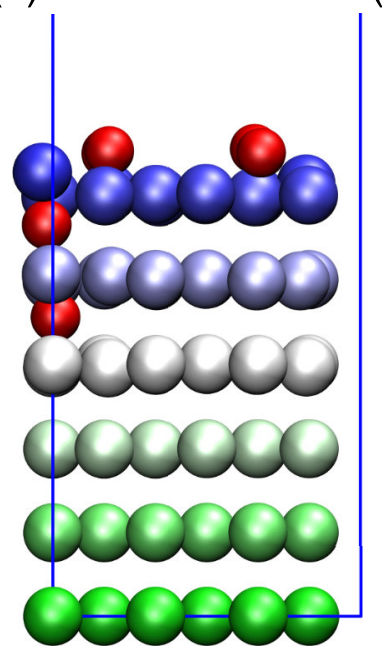

(b)

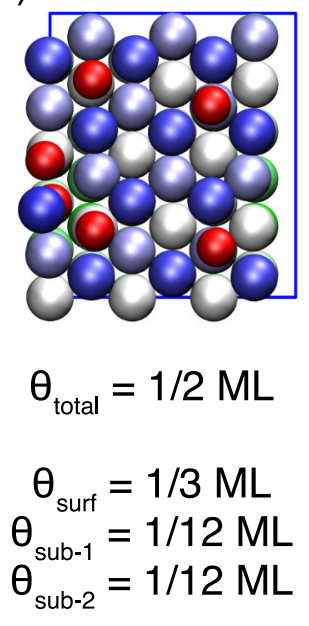

FIG. 6. (a) Side and (b) top views of a total oxygen coverage of 1 ML divided into $\frac{1}{3}$ ML on the surface, $\frac{1}{12} \mathrm{ML}$ in the first subsurface, and $\frac{1}{12} \mathrm{ML}$ in the second subsurface of $\mathrm{Ag}(111)$. The surface oxygen are uniformly distributed on the fcc hollow and hcp hollow sites and the subsurface oxygen are bound to octahedral sites. The color scheme is the same as that used in Figure 1 .

in Figure 5 are the same as those in Figure 2. The CBE values are plotted as shifts with respect to the CBE of $\theta_{\text {total }}=\frac{1}{12} \mathrm{ML}$ at the fcc hollow site. Results show that the CBE of surface oxygen increases up to $\theta_{\text {total }}=\frac{1}{3} \mathrm{ML}$ and subsequently decreases up to $\theta_{\text {total }}=\frac{7}{12}$ $\mathrm{ML}$, appearing to approach invariance with coverage. In contrast, the CBE of oxygen in the first and second subsurface regions more or less steadily decreases with coverage over the studied range. Further, the CBE of a total coverage of subsurface oxygen is greater than the $\mathrm{CBE}$ of the corresponding total coverage of surface oxygen, showing a gap of up to $0.8 \mathrm{eV}$ at the lowest coverage, whereas the $\mathrm{CBE}$ of oxygen atoms in the two subsurface regions are similar to each other at all coverages. As total coverage increases and more oxygen atoms share the same number of $\mathrm{Ag}$ atoms, the individual O-Ag interactions weaken, resulting in greater shielding of the core electrons by the valence electrons, and consequently lower $\mathrm{CBE}$ values.

Since each coverage studied in Figures 2 and 5 was constructed by populating a single type of binding site, the CBE shifts in Figure 5 do not include the effects of oxygen atoms co-adsorbed on different types of sites within the same surface or subsurface region or across two regions. Therefore, we calculated the CBE of oxygen atoms in the supercell shown in 
Figure 6 using DFT. The total coverage in the supercell was $\frac{1}{2} \mathrm{ML}$, divided into $\frac{1}{3} \mathrm{ML}$ on the surface, $\frac{1}{12} \mathrm{ML}$ in the first subsurface, and $\frac{1}{12} \mathrm{ML}$ in the second subsurface. The regional coverages were chosen to be close to the predicted Boltzmann-averaged coverages of 0.27 ML on the surface, 0.11 ML in the first subsurface, and 0.12 ML in the second subsurface, shown in Figure 3. The surface oxygen atoms were distributed equally between fcc hollow and hcp hollow sites, as predicted by the model, and the subsurface oxygen atoms were adsorbed to octahedral sites. We note that the average surface adsorption energy at $\frac{1}{3} \mathrm{ML}$ using this geometry but without any subsurface oxygen was calculated to be $3.40 \mathrm{eV}$ from both DFT and the model, in close agreement to the measured value of $3.44 \mathrm{eV}$ at $0.375 \mathrm{ML}$ by Campbell. $\stackrel{30}{.}$

The calculated shifts with respect to the CBE of $\theta_{\text {total }}=\frac{1}{12} \mathrm{ML}$ at the fcc hollow site, shown in Figure 7, reveal significant qualitative differences between the electronic properties of co-adsorbed surface and subsurface oxygen. First, the CBE of surface oxygen is similar to the corresponding value in the absence of subsurface oxygen, indicating that subsurface oxygen has a small impact on the CBE of surface oxygen. Second, the CBE of subsurface oxygen undergoes a large change in the presence of surface oxygen, such that the CBE gap between subsurface and surface oxygen increases from $+0.45-0.50 \mathrm{eV}$, when present independently, to $+0.85 \mathrm{eV}$ when present together. This change suggests that surface oxygen has a considerable effect on the CBE of subsurface oxygen, resulting in significantly different electronic properties of atomic oxygen adsorbed to the two regions. The calculated CBE gap is in good agreement with the measured CBE gap between the adsorbed oxygen species at low and high coverages measured in multiple XPS studies in which the species at high

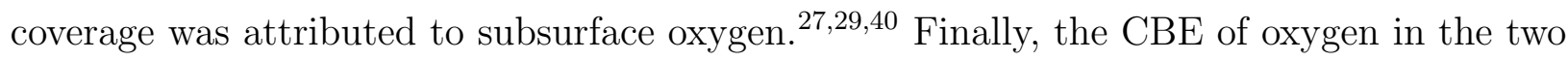
subsurface regions remain similar to each other in the presence or absence of surface oxygen. We note that the qualitative results are robust with change in arrangement of the oxygen atoms in each region. For example, when the oxygen atom in the second subsurface was separated from the oxygen atom in the first subsurface by a third-neighbor instead of the first-neighbor interaction shown in Figure 6, the CBE shift of oxygen on the surface, first subsurface, and second subsurface changed to $0.40 \mathrm{eV}, 1.45 \mathrm{eV}$, and $1.33 \mathrm{eV}$, respectively.

In the final step of our study, we calculated the projected density of states (PDOS) of the adsorbed oxygen atoms and their neighboring silver atoms in the optimized geometry shown in Figure 6 to investigate the origin of the CBE difference between surface and subsurface 


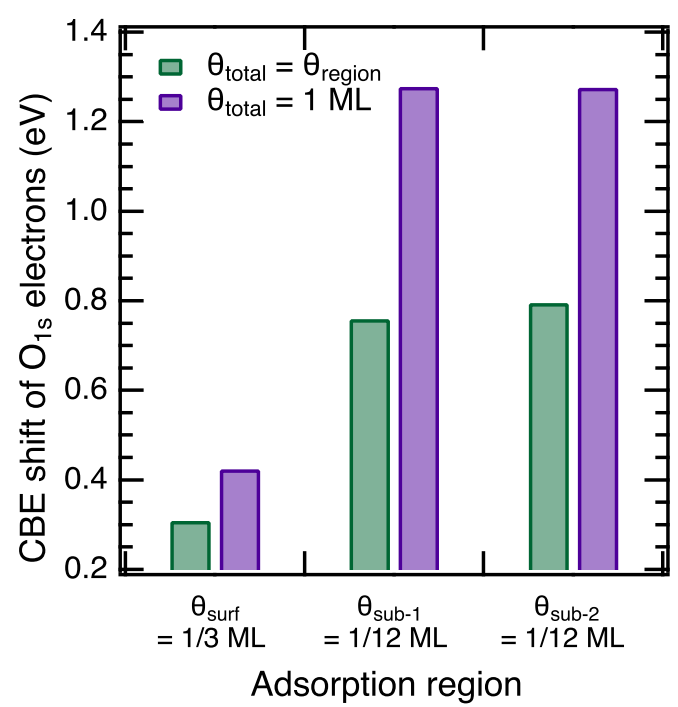

FIG. 7. Purple bars: CBE shift of 1 s electrons of $\mathrm{O}$ atoms at $\theta_{\text {total }}=1 \mathrm{ML}$ in the optimized geometry shown in Figure 6. The three bars show the CBE shifts of oxygen atoms in the surface ("surf"), first subsurface ("sub-1"), and second subsurface ("sub-2") of $\operatorname{Ag}(111)$, respectively. Green bars: CBE shift of $\mathrm{O}$ 1s electrons in the three reference systems, each with oxygen in a single region, and with the same regional oxygen coverage and arrangement as in the optimized geometry in (a).

oxygen. Figure 8(a) shows the PDOS of the valence orbitals of an oxygen adsorbed on an fcc hollow site (dotted lines) and the $4 \mathrm{~d}$ orbitals of a silver atom below it in the first surface layer (solid lines). The silver atom occupies one of the vertices of the three-fold hollow below the oxygen. Figure 8(b) shows the valence orbitals of the oxygen and the 5s-5p orbitals of the same silver atom. The spin-down electrons have the same PDOS as the spin-up electrons and are therefore omitted for clarity. The energies of the states are reported with respect to the Fermi energy of the system. Results show that the energy width of the valence oxygen orbitals in the oxygen-silver bonding region ranges from approximately $-5.65 \mathrm{eV}$ to $-2.5 \mathrm{eV}$, and contains a negligible contribution from the $2 \mathrm{~s}$ orbital. The antibonding region begins from approximately $-2.5 \mathrm{eV}$ and extends beyond $0 \mathrm{eV}$ into the unoccupied states of the band. The $4 \mathrm{~d}$ orbitals of $\mathrm{Ag}$ extend from $-6.0 \mathrm{eV}$ to $-2.5 \mathrm{eV}$ in the bonding region and make a negligible contribution to the antibonding region, whereas the 5 s orbitals extend from -6.5 $\mathrm{eV}$ to $-2.5 \mathrm{eV}$ in the bonding region and make a minor contribution to the antibonding region.

The PDOS plots in Figure 8 show a broad overlap between $\mathrm{O} 2 \mathrm{p}$ orbitals and $\mathrm{Ag} 4 \mathrm{~d}$ 

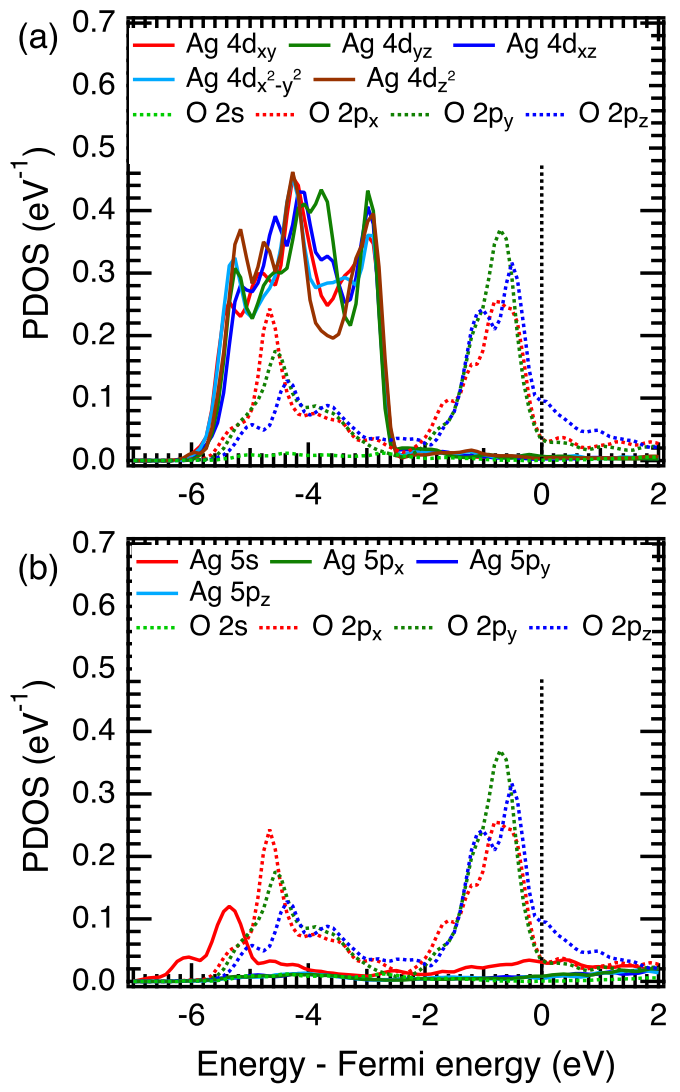

FIG. 8. PDOS plots of an oxygen atom adsorbed on an fcc hollow site of $\mathrm{Ag}(111)$ from the optimized geometry shown in Figure 6. (a) $2 \mathrm{~s}$ and $2 \mathrm{p}$ of the oxygen atom and the $4 \mathrm{~d}$ orbitals of a silver atom below it in the first surface layer, (b) $2 \mathrm{~s}$ and $2 \mathrm{p}$ of the oxygen atom and the $5 \mathrm{~s}$ and $5 \mathrm{p}$ orbitals of the same silver atom.

orbitals, and a weak overlap between $\mathrm{O} 2 \mathrm{p}$ orbitals and $\mathrm{Ag} 5 \mathrm{~s}$ orbitals in the bonding region. The analogous PDOS plot in Figure 9 for an oxygen atom in the octahedral site of the first subsurface and a silver atom below it in the second layer shows that $\mathrm{O} 2 \mathrm{p}$ orbitals drop to lower energy in the bonding region, ranging from approximately $-6.25 \mathrm{eV}$ to $-2.5 \mathrm{eV}$. The $\mathrm{Ag}$ $4 \mathrm{~d}$ orbitals also drop lower to $-6.25 \mathrm{eV}$, whereas the $\mathrm{Ag} 5$ s orbitals remain within an energy range of $-6.5 \mathrm{eV}$ to $-2.5 \mathrm{eV}$, similar to that of the $\mathrm{Ag}$ atom in the first surface layer. The $\mathrm{O}$ $2 \mathrm{p}$ orbitals show a relatively narrow overlap with the left edge of the Ag $4 \mathrm{~d}$ orbitals and a stronger overlap with the Ag 5s orbital compared to the O-Ag interaction on the surface.

The $\mathrm{O} 2 \mathrm{p}$ orbitals and $\mathrm{Ag} 5 \mathrm{~s}$ orbitals in the second subsurface (Figure 10) drop to even lower energy in the bonding region, starting from $-6.5 \mathrm{eV}$ and $-6.8 \mathrm{eV}$, respectively, whereas 

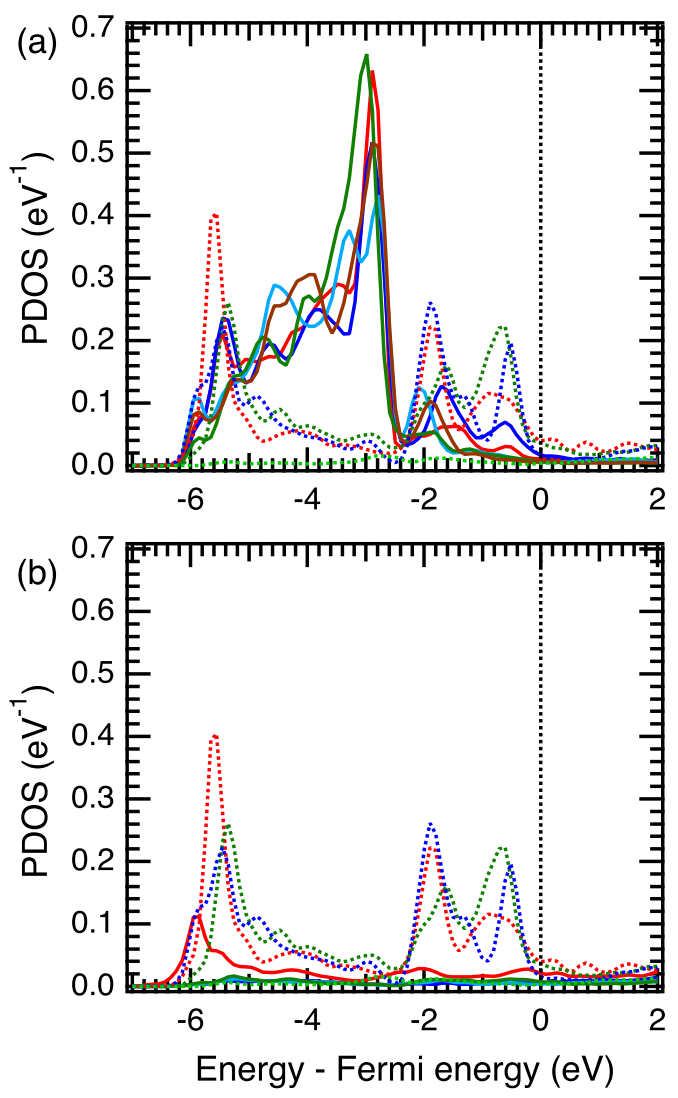

FIG. 9. PDOS plots of an oxygen atom adsorbed to an octahedral site in the first subsurface of $\operatorname{Ag}(111)$ from the optimized geometry shown in Figure 6. (a) $2 \mathrm{~s}$ and $2 \mathrm{p}$ of the oxygen atom and the $4 \mathrm{~d}$ orbitals of a silver atom below it in the second surface layer, (b) $2 \mathrm{~s}$ and $2 \mathrm{p}$ of the oxygen atom and the $5 \mathrm{~s}$ and $5 \mathrm{p}$ orbitals of the same silver atom.

the Ag 4d orbitals remain in the same energy range as the Ag 4d orbitals in the first subsurface (Figure 9p. These changes lead to weaker overlap between $\mathrm{O} 2 \mathrm{p}$ and $\mathrm{Ag} 4 \mathrm{~d}$, but stronger overlap between $\mathrm{O} 2 \mathrm{p}$ and $\mathrm{Ag} 5$ s compared to the first subsurface.

Overall, the PDOS plots show that the $\mathrm{O}$ and $\mathrm{Ag}$ orbitals are energetically more stable in the subsurface regions compared to the surface region. Further, the energy alignment between the $\mathrm{Ag}$ and $\mathrm{O}$ orbitals changes between the surface and subsurface, due to which the $\mathrm{O} 2 \mathrm{p}$ orbitals overlap primarily with $\mathrm{Ag} 4 \mathrm{~d}$ orbitals on the surface, but with both $\mathrm{Ag}$ $4 \mathrm{~d}$ and $5 \mathrm{~s}$ orbitals in the subsurface. The overlap with $\mathrm{O} 2 \mathrm{p}$ and $\mathrm{Ag} 5 \mathrm{~s}$ is greater in the second subsurface than in the first subsurface. The qualitative differences in $\mathrm{O}-\mathrm{Ag}$ bonding between the surface and the subsurface result in significantly greater CBE of subsurface 

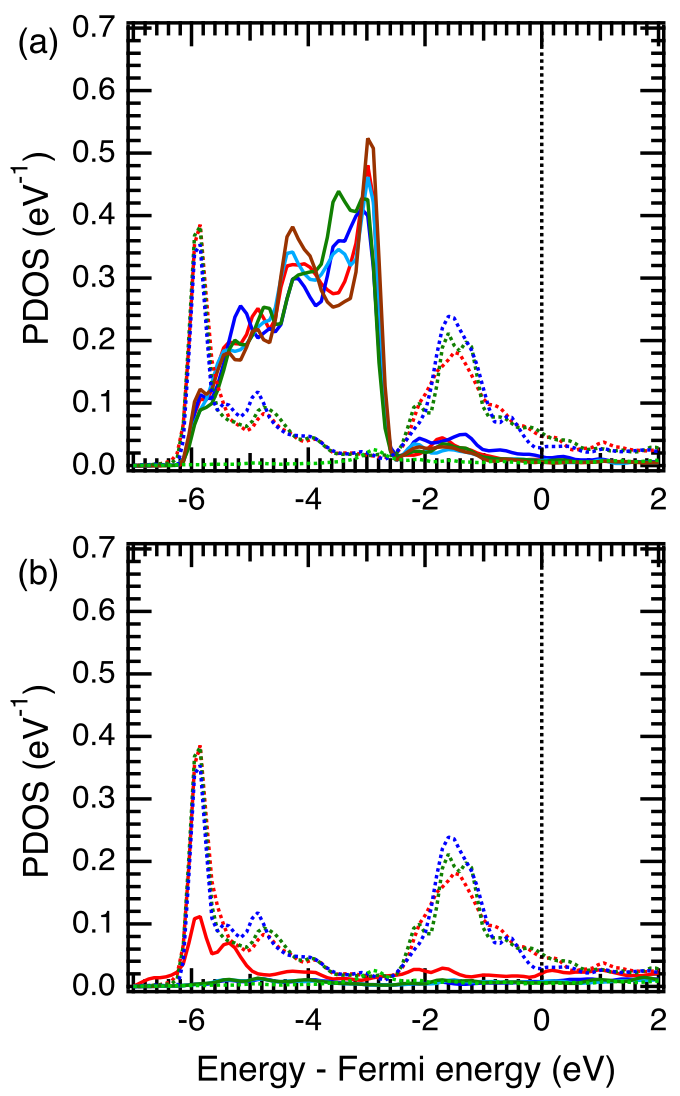

FIG. 10. PDOS plots of an oxygen atom adsorbed to an octahedral site in the second subsurface of $\operatorname{Ag}(111)$ from the optimized geometry shown in Figure 6. (a) 2 s and $2 p$ of the oxygen atom and the $4 \mathrm{~d}$ orbitals of a silver atom below it in the third surface layer, (b) $2 \mathrm{~s}$ and $2 \mathrm{p}$ of the oxygen atom and the $5 \mathrm{~s}$ and $5 \mathrm{p}$ orbitals of the same silver atom.

oxygen compared to surface oxygen. Importantly, the increasing overlap between $\mathrm{O} 2 \mathrm{p}$ and $\mathrm{Ag} 5 \mathrm{~s}$ in the subsurface regions screens the oxygen from neighboring oxygen atoms and decreases the inter-oxygen electrostatic repulsion, resulting in attractive or cooperative first-neighbor O-O interactions in the subsurface regions. We note that the CBE and PDOS of other surface oxygen atoms from Figure 6 were qualitatively similar to the plots shown in Figures 7 and 8 , and the same trend in orbital stabilization and alignment was found when we considered silver atoms above instead of below the subsurface oxygen atoms. Together, the CBE and PDOS results highlight the qualitative differences in electronic properties and chemical bonding of atomic oxygen between the surface and subsurface of $\mathrm{Ag}(111)$. 


\section{CONCLUSIONS}

In summary, we have developed a lattice-gas model that includes both surface and subsurface adsorption sites in a crystalline solid and describes the effects of adsorbate coverage using pairwise interactions between co-adsorbates. We have parametrized the model using DFT to study the competition between surface and subsurface adsorption of atomic oxygen on the $\operatorname{Ag}(111)$ surface as a function of increasing coverage. Our study shows that oxygen is likely to occupy the subsurface of $\mathrm{Ag}(111)$ at total coverages greater than $1 / 4 \mathrm{ML}$ and prefers to occupy the second subsurface rather than the first subsurface at coverages greater than 1/2 ML. The stronger overlap between the $2 \mathrm{p}$ orbitals of oxygen and $5 \mathrm{~s}$ orbital of silver in the subsurface screens the oxygen atoms from each other more effectively, resulting in less inter-oxygen repulsion and consequent accumulation of oxygen in the subsurface. This result is manifested in the model via the transformation in first-neighbor $\mathrm{O}-\mathrm{O}$ interaction energies from strongly repulsive on the surface to weakly attractive in the first subsurface, to moderately attractive in the second subsurface. The model shows that the subsurface of the metal acts as a reservoir of oxygen, and the qualitative differences in oxygen-silver bonding between the surface and subsurface suggest that the two species could play distinct roles in

surface chemistry. Future work will apply the model to perform Monte Carlo simulations to explore the effects of surface temperature and desorption on the interplay between surface and subsurface adsorption.

\section{SUPPLEMENTARY MATERIAL}

See supplementary information for images of surface sites, parameters of the adsorption model, and a parity plot comparing the model to DFT.

\section{ACKNOWLEDGMENTS}

This research was supported by the National Science Foundation CAREER grant CHE1753273. Coverage-dependent DFT calculations in this work used the Stampede cluster at the Texas Advanced Computing Center, the Bridges cluster at the Pittsburgh Supercomputing Center, and the Comet cluster at the San Diego Supercomputer Center through the startup allocation CHE180046 at the Extreme Science and Engineering Discovery Environment (XSEDE). XSEDE is supported by National Science Foundation grant number 
ACI-1548562. Computations of the adsorption model were performed on the computational resources at the Infrastructure for Scientific Applications and Advanced Computing (ISAAC) supported by the University of Tennessee. SR thanks Daniel Killelea for helpful discussions.

DATA AVAILABILITY STATEMENT The data that supports the findings of this study are available within the article and its supplementary material.

\section{REFERENCES}

${ }^{1}$ J. M. Sanchez, F. Ducastelle, and G. Gratias, Physica A 128, 334 (1984),

${ }^{2}$ J. M. Sanchez, Phys. Rev. B 48, 14013 (1993).

${ }^{3}$ J. M. Sanchez, Phys. Rev. B 81, 13 (2010).

${ }^{4}$ J. M. Sanchez, J. Phase Equilib. Diff. 38, 238 (2017).

${ }^{5}$ G. Collinge, K. Groden, C. Stampfl, and J.-S. McEwen, J. Phys. Chem. C 124, 2923 (2019).

${ }^{6}$ A. J. R. Hensley, G. Collinge, Y. Wang, and J.-S. McEwen, J. Phys. Chem. C 124, 7254 $(2020)$.

${ }^{7}$ D. J. Schmidt, W. Chen, C. Wolverton, and W. F. Schneider, J. Chem. Theory Comput. 8, 264 (2012).

${ }^{8}$ L. Tan, L. Huang, Y. Liu, and Q. Wang, Langmuir 34, 5174 (2018).

${ }^{9}$ F. O. Sanchez-Varretti, E. d. V. Gómez, L. B. Avalle, F. M. Bulnes, M. C. Gimenez, and A. J. Ramirez-Pastor, Appl. Surf. Sci. 500 (2020).

${ }^{10} \mathrm{M}$. Huš and A. Hellman, ACS Catal. 9, 1183 (2018).

${ }^{11}$ J. W. Chen, H. X. Chen, T. W. Yu, R. C. Li, Y. Wang, Z. P. Shao, and S. Q. Song, Electrochem. Energ. Rev. (2021).

${ }^{12}$ J. Greeley, J. K. Norskov, and M. Mavrikakis, Annu. Rev. Phys. Chem. 53, 319 (2002).

${ }^{13}$ N. Kenge, S. Pitale, and K. Joshi, Surf. Sci. 679, 188 (2019).

${ }^{14}$ F. B. de Mongeot, U. Valbusa, and M. Rocca, Surf. Sci. 339, 291 (1995).

${ }^{15}$ M. L. Bocquet, A. Michaelides, P. Sautet, and D. A. King, Phys. Rev. B 68 (2003).

${ }^{16}$ A. Michaelides, M. L. Bocquet, P. Sautet, A. Alavi, and D. A. King, Chem. Phys. Lett. 367, 344 (2003).

${ }^{17}$ A. Michaelides, K. Reuter, and M. Scheffler, J. Vac. Sci. Technol. A 23, 1487 (2005).

${ }^{18}$ Y. Xu, J. Greeley, and M. Mavrikakis, J. Am. Chem. Soc. 127, 12823 (2005). 
${ }^{19}$ T. Pu, H. Tian, M. E. Ford, S. Rangarajan, and I. E. Wachs, ACS Catal. 9, 10727 (2019). ${ }^{20}$ T. E. Jones, R. Wyrwich, S. Böcklein, T. C. R. Rocha, E. A. Carbonio, A. Knop-Gericke, R. Schlögl, S. Günther, J. Wintterlin, and S. Piccinin, J. Phys. Chem. C 120, 28630 (2016).

${ }^{21}$ T. E. Jones, R. Wyrwich, S. Böcklein, E. A. Carbonio, M. T. Greiner, A. Y. Klyushin, W. Moritz, A. Locatelli, T. O. Menteş, M. A. Niño, A. Knop-Gericke, R. Schlögl, S. Günther, J. Wintterlin, and S. Piccinin, ACS Catal. 8, 3844 (2018).

${ }^{22}$ V. I. Avdeev and G. M. Zhidomirov, Surf. Sci. 492, 137 (2001).

${ }^{23}$ S. Linic and P. Christopher, Chemcatchem 2, 1061 (2010).

${ }^{24}$ M. Ozbek, I. Onal, and R. A. van Santen, J. Catal. 284, 230 (2011).

${ }^{25}$ P. Christopher and S. Linic, J. Am. Chem. Soc. 130, 11264 (2008).

${ }^{26}$ J. Derouin, R. G. Farber, M. E. Turano, E. V. Iski, and D. R. Killelea, ACS Catal. 6, 4640 (2016).

${ }^{27}$ M. E. Turano, R. G. Farber, E. C. N. Oskorep, R. A. Rosenberg, and D. R. Killelea, J. Phys. Chem. C 124, 1382 (2019).

${ }^{28}$ X. Bao, J. V. Barth, G. Lehmpfuhl, R. Schuster, Y. Uchida, R. Schlögl, and G. Ertl, Surf. Sci. 284, 14 (1993).

${ }^{29}$ O. G. Ashkhotov, S. A. Khubezhov, and I. B. Ashkhotova, Semiconductors 53, 1983 $(2020)$.

${ }^{30}$ C. T. Campbell, Surf. Sci. 157, 43 (1985).

${ }^{31}$ C. Heine, B. Eren, B. A. J. Lechner, and M. Salmeron, Surf. Sci. 652, 51 (2016).

${ }^{32}$ S. Bocklein, S. Gunther, and J. Wintterlin, Angew. Chem. Int. Ed. 52, 5518 (2013).

${ }^{33}$ A. J. Garza, A. T. Bell, and M. Head-Gordon, J. Phys. Chem. Lett. 9, 601 (2018).

${ }^{34}$ S. T. Ceyer, Acc. Chem. Res. 34, 737 (2001).

${ }^{35}$ A. Kiejna and B. I. Lundqvist, Phys. Rev. B 63 (2001).

${ }^{36}$ M. Todorova, W. X. Li, M. V. Ganduglia-Pirovano, C. Stampfl, K. Reuter, and M. Scheffler, Phys. Rev. Lett. 89, 096103 (2002).

${ }^{37}$ W.-X. Li, C. Stampfl, and M. Scheffler, Phys. Rev. B 65 (2002).

${ }^{38}$ W.-X. Li, C. Stampfl, and M. Scheffler, Phys. Rev. B 67 (2003).

${ }^{39}$ W.-X. Li, C. Stampfl, and M. Scheffler, Phys. Rev. Lett. 90, 256102 (2003).

${ }^{40}$ T. E. Jones, T. C. Rocha, A. Knop-Gericke, C. Stampfl, R. Schlogl, and S. Piccinin, Phys. Chem. Chem. Phys. 17, 9288 (2015). 
${ }^{41}$ T. E. Jones, T. C. R. Rocha, A. Knop-Gericke, C. Stampfl, R. Schlögl, and S. Piccinin, ACS Catal. 5, 5846 (2015).

${ }^{42}$ J. Greeley and M. Mavrikakis, J. Phys. Chem. C 111, 7992 (2007).

${ }^{43}$ E. A. Carbonio, T. C. R. Rocha, A. Y. Klyushin, I. Pis, E. Magnano, S. Nappini, S. Piccinin, A. Knop-Gericke, R. Schlogl, and T. E. Jones, Chem. Sci. 9, 990 (2018).

${ }^{44}$ B. W. J. Chen, D. Kirvassilis, Y. Bai, and M. Mavrikakis, J. Phys. Chem. 123, 7551 (2019).

${ }^{45}$ S. B. Isbill, S. Roy, and D. J. Keffer, Mol. Simulat. 43, 355 (2017).

${ }^{46}$ J. P. Perdew, K. Burke, and M. Ernzerhof, Phys. Rev. Lett. 77, 3865 (1996).

${ }^{47}$ J. P. Perdew, K. Burke, and M. Ernzerhof, Phys. Rev. Lett. 78, 1396 (1997).

${ }^{48}$ B. Hammer, L. B. Hansen, and J. K. Nørskov, Phys. Rev. B 59, 7413 (1999).

${ }^{49}$ G. Kresse and J. Hafner, Phys. Rev. B 47, 558 (1993).

${ }^{50}$ G. Kresse and J. Hafner, Phys. Rev. B 49, 14251 (1994).

${ }^{51}$ G. Kresse and J. Furthmüller, Phys. Rev. B 54, 11169 (1996).

${ }^{52}$ G. Kresse and F. J., Comp. Mater. Sci. 6, 15 (1996).

${ }^{53}$ P. E. Blochl, Phys. Rev. B 50, 17953 (1994).

${ }^{54}$ G. Kresse and D. Jouber, Phys. Rev. B 59, 1758 (1999).

${ }^{55}$ M. Methfessel and A. T. Paxton, Phys. Rev. B 40, 3616 (1989).

${ }^{56}$ H. J. Monkhorst and J. D. Pack, Phys. Rev. B 13, 5188 (1976).

${ }^{57}$ N. Ashcroft and N. Mermin, Solid State Physics, 1st ed. (Saunders College Publishing, Belmount, 1976) p. 833.

${ }^{58}$ G. Henkelman, A. Arnaldsson, and H. Jónsson, Comp. Mater. Sci. 36, 354 (2006).

${ }^{59}$ E. Sanville, S. D. Kenny, R. Smith, and G. Henkelman, J. Comput. Chem. 28, 899 (2007).

${ }^{60}$ W. Tang, E. Sanville, and G. Henkelman, J. Phys. Condens. Matter 21, 084204 (2009).

${ }^{61}$ L. Köhler and G. Kresse, Phys. Rev. B 70, 165405 (2004).

${ }^{62}$ P. Janthon, S. M. Kozlov, F. Vines, J. Limtrakul, and F. Illas, J. Chem. Theory Comput. 9, 1631 (2013).

${ }^{63}$ N. Pueyo Bellafont, F. Vines, W. Hieringer, and F. Illas, J. Comput. Chem. 38, 518 $(2017)$. 